lesions. The primary outcome was the completion rate of the self-completion ESD method. The secondary outcomes were the procedure time, en-bloc resection rate, perforation rate, and number of injections during the procedure.

Results In this study, all 12 cases of ESD were completed using the self-completion method (completion rate: 100\%). The median procedure time (range) was 483.5 (276-936) seconds [median incision time; 240.4 (98-605) seconds and median dissection time; 222 (137-617) seconds]. En-bloc and complete resection rates were $100 \%$ each. No perforation was noted during any of the procedures. The median number of injections was 10.5 (4-15). The procedure time significantly decreased with experience $(\mathrm{P}=0.0199)$.

Conclusions The self-completion ESD method using only one Endosaber without any assistance achieved a 100\% en-bloc resection rate without any perforation. Reduction in the need for an additional device or assistance was successful. This method may contribute to reducing the total cost of ESD procedure.

\section{IDDF2019-ABS-0118 ELECTROACUPUNCTURE PLUS ON-DEMAND GASTROCAINE FOR REFRACTORY FUNCTIONAL DYSPEPSIA: PRAGMATIC RANDOMIZED TRIAL}

${ }^{1}$ Vincent Chi Ho Chung ${ }^{*},{ }^{2}$ Charlene Hoi Lam Wong, ${ }^{3}$ Irene Xin Yin Wu, ${ }^{2}$ Jessica Yuet Ling Ching, 'William Kwok Wai Cheung, 'Benjamin Hon Kei Yip, ${ }^{4}$ Kam Leung Chan, ${ }^{2}$ Pui Kuan Cheong, ${ }^{2}$ Justin Che Yuen Wu. 'Jockey Club School of Public Health and Primary Care, The Chinese University of Hong Kong, Hong Kong; ${ }^{2}$ Department of Medicine and Therapeutics, The Chinese University of Hong Kong, Hong Kong; ${ }^{3}$ Xiang-Ya School of Public Health, Central South University, Chang-Sha, Hu-Nan, China; ${ }^{4}$ School of Chinese Medicine, The Chinese University of Hong Kong, Hong Kong

\subsection{6/gutjnl-2019-IDDFabstracts.156}

Background Treatment options for functional dyspepsia (FD) refractory to pharmacological treatments are limited, but the effectiveness of electroacupuncture (EA) is uncertain.

Our aim was to assess the effectiveness of EA combined with on-demand gastrocaine.

Methods We conducted a single-centre, assessor-blind, randomized parallel-group 2-arm trial on $H$. pylori negative FD patients of the postprandial distress syndrome (PDS) subtype refractory to proton pump inhibitor, prokinetics or $\mathrm{H}_{2}$ antagonists. Enrolled participants were block randomized in a 1:1 ratio, with concealed random sequence. The treatment and control groups both received on-demand gastrocaine for 12 weeks, but only those in treatment group were offered 20 sessions of EA over 10 weeks. The primary endpoint was the between-group difference in the proportion of patients achieving adequate relief of symptoms at week 12 .

Results Of 132 participants randomly assigned to EA plus ondemand gastrocaine $(n=66)$ or on-demand gastrocaine alone $(n=66), 125(94.7 \%)$ completed all follow-up at 12 weeks. The EA group had a compliance rate $97.7 \%$. They had a significantly higher likelihood in achieving adequate symptom relief at 12 weeks, with a clinically relevant number needed to treat (NNT) value of 2.36 (95\%CI: 1.74-3.64). Among secondary outcomes, statistically and clinically significant improvements were observed among global symptom $(\mathrm{NNT}=3.85(95 \% \mathrm{CI}: 2.63,7.69))$; postprandial fullness and early satiation ( $\mathrm{NNT}=5.00 \quad(95 \% \mathrm{CI}: 2.86,25.00))$; as well as epigastric pain, epigastric burning and postprandial nausea $(\mathrm{NNT}=4.17$ (95\%CI: 2.56, 11.11)). Adverse events were minimal and non-significant.

Conclusions For refractory FD, EA provides significant, clinically relevant symptom relief when added to on-demand gastrocaine. (ChiCTR-IPC-15007109)

\section{IDDF2019-ABS-0119 ENDOSCOPIC ULTRASOUND-GUIDED LEFT LOBE LIVER LESIONS BIOPSY (EUS-LLB) WITH ATYPICAL MALIGNANCIES, AN ALTERNATIVE APPROACH TO RADIOLOGICAL IMAGE-GUIDED BIOPSY}

Adeel Urrehman*, Mohammad Mubarak. Sindh Institute of Urology and Transplantation, Pakistan

\subsection{6/gutjnl-2019-IDDFabstracts.157}

Background Endoscopic ultrasound (EUS) is gaining attraction as an alternative method of biopsy. It offers a more targeted approach for focal lesions in liver especially those areas which are accessible via EUS-guided method.

Methods A total of 7 patients from Feb 2018 till September 2018 underwent EUS-guided Left Lobe lesions biopsies. EUS Guided LLB performed using 22G FNA needle, 2 passes were done with slow pull technique over one minute with 10-15 strokes in each pass to obtained core samples. Duration of procedures ranges from 15-30 minutes.

Results Case 1, 78-Years-old male with a history of gastrectomy $\underline{5}$ years back for biopsy-proven gastric cancer, presented with weight loss and vague epigastric pain, CT scan showed left lobe SOL. Biopsy showed well-differentiated Adenocarcinoma from GI Tract. Case 2, 58-years-old male presented with weight loss and Liver mass on CT Scan. Hepatitis B \& C screen was negative with normal AFP levels. Biopsy revealed Sarcomatoid Carcinoma. Case 3, 53-Years-old male presented with weight loss and abdominal pain. He had multiple lesions identified on CT scan in the liver. Biopsy revealed Neuroendocrine Tumor. Case 4, 35-years-old male presented with abdominal pain. Laboratory tests showed antiHCV reactive with normal AFP levels. CT Scan showed liver lesion suggestive of atypical Hepatocellular carcinoma. Biopsy revealed Smooth Muscle Tumor. As part of workup, he also had gastroscopy and Colonoscopy with no evidence of luminal malignancy. Case 5, 60-years-old female presented with Weight loss, CT scan showed pancreatic malignancy with liver metastasis. Biopsy revealed Metastatic Adenocarcinoma. Case 6, 42-years-old female presented with Obstructive jaundice from Ampullary Carcinoma. She underwent EUS staging which revealed left lobe lesion. Biopsy revealed Metastatic Adenocarcinoma. Case 7, 32-years-old male presented with Weight loss and Obstructive jaundice, CT scan showed left lobe malignancy consistent with Cholangiocarcinoma with normal AFP and CA19-9 levels, his hepatitis B \& C screen negative, the biopsy revealed Lymphoproliferative disease (Lymphoma).

Conclusions EUS-guided LLB is an alternative new technique for biopsy of liver lesions with suspected atypical malignancies. 